\title{
Recovering from a pandemic: pulmonary fibrosis after SARS-CoV-2 infection
}

\author{
Ruben J. Mylvaganam $\mathbb{\circledR}^{1}$, Joseph I. Bailey ${ }^{1}$, Jacob I. Sznajder ${ }^{1}$ and Marc A. Sala ${ }^{1}$, on behalf of the \\ Northwestern Comprehensive COVID Center Consortium ${ }^{2}$
}

${ }^{1}$ Division of Pulmonary and Critical Care Medicine, Feinberg School of Medicine, Northwestern University, Chicago, IL, USA. ${ }^{2}$ A full list of the Northwestern Comprehensive COVID Center Consortium members can be found in the Acknowledgements section.

Corresponding author: Ruben J. Mylvaganam (ruben.mylvaganam@northwestern.edu)

Shareable abstract (@ERSpublications)

A complication of COVID-19, PASC-pulmonary fibrosis, has the potential to become a global respiratory health crisis. Dedicated surveillance, mechanistic understanding and clinical and research efforts are needed to confront this emerging sequela of COVID-19. https://bit.ly/3GEyB04

Cite this article as: Mylvaganam RJ, Bailey JI, Sznajder JI, et al. Recovering from a pandemic: pulmonary fibrosis after SARS-CoV-2 infection. Eur Respir Rev 2021; 30: 210194 [DOI: 10.1183/ 16000617.0194-2021].

Copyright @The authors 2021

This version is distributed under the terms of the Creative Commons Attribution NonCommercial Licence 4.0. For commercial reproduction rights and permissions contact permissions@ersnet.org

Received: 23 Aug 2021

Accepted: 24 Oct 2021

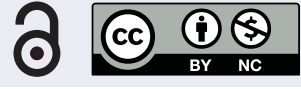

\section{Abstract}

Acute manifestations of SARS-CoV-2 infection continue to impact the lives of many across the world. Post-acute sequelae of coronavirus disease 2019 (COVID-19) may affect 10-30\% of survivors of COVID19, and post-acute sequelae of COVID-19 (PASC)-pulmonary fibrosis is a long-term outcome associated with major morbidity. Data from prior coronavirus outbreaks (severe acute respiratory syndrome and Middle East respiratory syndrome) suggest that pulmonary fibrosis will contribute to long-term respiratory morbidity, suggesting that PASC-pulmonary fibrosis should be thoroughly screened for through pulmonary function testing and cross-sectional imaging. As data accumulates on the unique pathobiologic mechanisms underlying critical COVID-19, a focus on corollaries to the subacute and chronic profibrotic phenotype must be sought as well. Key aspects of acute COVID-19 pathobiology that may account for increased rates of pulmonary fibrosis include monocyte/macrophage-T-cell circuits, profibrotic RNA transcriptomics, protracted elevated levels of inflammatory cytokines, and duration of illness and ventilation. Mechanistic understanding of PASC-pulmonary fibrosis will be central in determining therapeutic options and will ultimately play a role in transplant considerations. Well-designed cohort studies and prospective clinical registries are needed. Clinicians, researchers and healthcare systems must actively address this complication of PASC to minimise disability, maximise quality of life and confront a post-COVID-19 global health crisis.

\section{Introduction}

The outbreak of the novel severe acute respiratory syndrome coronavirus 2 (SARS-CoV-2) responsible for coronavirus disease 2019 (COVID-19) was first reported on 31 December 2019 in the Hubei province in China. Since that time, SARS-CoV-2 has spread globally with a devastating impact on both the health and economies of most nations. The World Health Organization (WHO) reports that as of 1 October 2021 there have been approximately 232 million confirmed cases and 4.8 million deaths from COVID-19 globally. As of the same date, in North America, there have been 47 million confirmed cases and 984000 deaths. These North American estimates represent about 20\% of the global case and mortality burden, despite North America having about 5\% of the total world's population. The mortality figures fail to capture the impact of the infection on the 40 million individuals in North America at risk for the development of post-acute sequelae of COVID-19 (PASC), which by some estimates, affects 10-30\% survivors of COVID-19 [1]. PASC has been defined as persistent symptoms and/or long-term complications of SARS-CoV-2 more than 4 weeks after the initial onset of symptoms [2].

Two of the complications of COVID-19 that have the risk of causing post-acute sequelae are those of acute respiratory distress syndrome (ARDS) and COVID pneumonia [3, 4]. The likelihood of these complications are greater in those of older age, male sex, and with chronic comorbid medical conditions [3]. 
Evidence on the subacute and long-term sequelae of COVID-19 are accumulating. Pulmonary fibrosis is the most severe manifestation of post-infectious lung injury and has a profound long-term impact on patients' respiratory health. In addition to age and chronic comorbid conditions, the need for invasive or noninvasive mechanical ventilation, and female sex are some identified risk factors of respiratory PASC [2, 4].

In this review we present what has been published thus far to describe pulmonary fibrosis as a phenotype of PASC (hereafter referred to as PASC-pulmonary fibrosis) and discuss the implications for future discovery and treatment.

Pulmonary fibrosis in the context of SARS and MERS

The recent coronavirus outbreaks (the severe acute respiratory syndrome (SARS) epidemic of 2003 and the Middle East respiratory syndrome (MERS) outbreak of 2012) have demonstrated that persistent respiratory symptoms and radiographic abnormalities continue beyond the period of acute illness. At 6 months, 33 of 110 survivors of SARS showed persistent radiographic abnormalities and reductions in forced vital capacity (FVC), total lung capacity and transfer capacity [5]. A prospective 15-year study of healthcare workers infected with SARS during the 2003 epidemic revealed that radiographic changes that had not resolved within the first 2 years after acute infection tended to plateau and radiographic abnormalities correlated with continued impairment in diffusion capacity on lung function testing [6]. There are similar findings from studies that emerged from the MERS outbreak in 2012. DAs et al. [7] revealed persistent imaging abnormalities (ground-glass opacities) at 1-year post-acute infection in 55 individuals and showed that a chest radiographic score both correlated and was an independent predictor of mortality.

The ongoing nature of the COVID-19 pandemic and the lack of consensus with regard to screening and diagnosis for the spectrum of PASC phenotypes makes the reporting on the magnitude of PASC-pulmonary fibrosis incomplete. Given the genetic homology between SARS-CoV-2 with both SARS and MERS (79\% and 50\%, respectively), there may be a similar and possibly increased likelihood of long-term pulmonary complications after COVID-19 [8-10]. SARS-CoV-2 has infected greater than 5 orders of magnitude more patients than either SARS or MERS. Therefore, it is expected that the global burden of PASC-pulmonary fibrosis and subsequent respiratory morbidity will be of significant impact on patients and society.

Incidence and natural history

Pre-pandemic context

Prior to the COVID-19 pandemic, studies in the low-tidal volume ventilation era of ARDS suggested that between 20 and $40 \%$ of survivors of ARDS suffer persistent symptoms of breathlessness or reduced quality of life [11, 12]. In a study by WiLCox et al. [13], 20 of 24 (75\%) survivors of ARDS with 5-year follow-up high-resolution computed tomography (HRCT) images had abnormal radiographic findings. Initially, persistent radiographic abnormalities were only loosely correlated with worsened quality of life $[11,12]$. Subsequently, Burnham et al. [14] analysed prospective data of patients enrolled in a randomised control trial of acute lung injury and concluded that persistent HRCT abnormalities were correlated with worsened pulmonary-specific health-related quality of life as assessed by the St. George's Respiratory Questionnaire (SGRQ) surveys. This group also showed that reticular infiltrates 14 days after acute lung injury predicted poorer quality of life at 180 days, even while controlling for other potential covariates [14].

\section{COVID-19 pandemic context}

In the context of the COVID-19 pandemic, up to $40 \%$ of infected individuals may develop viral pneumonia and $20 \%$ of these cases can develop severe ARDS [15]. Early in the pandemic, investigators postulated that acute COVID-19 infection leads to an abnormal form of ARDS [16]. However, studies have demonstrated that patients with acute COVID-19 pneumonia requiring mechanical ventilation exhibit a similar clinical phenotype and respiratory mechanics to other forms of ARDS [17]. A key distinction is that, compared with patients mechanically ventilated for influenza pneumonia, patients intubated for respiratory failure from SARS-CoV-2 pneumonia have an approximately 3-fold longer duration of mechanical ventilation (14 days as compared with 4 days) [18]. Additional studies have reported a similar duration of mechanical ventilation and intensive care unit (ICU) stay [19-21]. In North America, reports from survivors of COVID-19 in the Michigan COVID-19 initiative (MI-COVID19) reported that among 488 participants who completed a follow-up survey, 33\% had persistent symptoms and 19\% had worsening symptoms. The most common symptom described was breathlessness while walking up stairs (23\%) [22]. Respiratory PASC symptoms are also reported in similar frequencies in Europe after 60 days and in China over a 6-month follow-up period [4, 23-25]. Among these studies, Huang and colleagues [4] reported that disease severity, requirement for invasive or noninvasive ventilation, and female sex were risk factors for the development of respiratory PASC symptoms. At this time, there is limited guidance to 
clinicians on the appropriate timing of follow-up, screening and imaging for individuals with persistent or new respiratory symptoms after COVID-19 infection.

\section{Screening for PASC-pulmonary fibrosis}

Pulmonary function testing

Screening studies of pulmonary function changes from the prior coronavirus outbreaks lend some background as to how to approach screening during the current COVID-19 pandemic. Pulmonary function testing (PFT) during the SARS and MERS outbreaks showed evidence of persistent limitations in diffusion capacity and restrictive physiology. The applicability of these types of studies are often confounded by the lack of baseline testing [26-28]. In the same light, there have been many reports suggesting impaired diffusion and restrictive physiology in lung function testing after acute COVID-19 [25, 29-31]. These reports have also been hindered by both a lack of baseline testing and nonuniformity in timing from acute illness of follow-up assessment. Acknowledging the limitations of heterogeneity in study design, lack of baseline testing and timing of assessments, a recent meta-analysis and systematic review by ToRRES-CASTRO et al. [32] found decreased diffusing capacity in 39\%, a restrictive ventilatory pattern in $15 \%$, and an obstructive ventilatory pattern in 7\% of COVID-19 survivors.

The British Thoracic Society recommends obtaining PFTs at 3 months post-discharge in all survivors of critical COVID-19 and recommends PFTs in those with mild to moderate disease if they have abnormal radiographic imaging [33]. Similar guidance is given by the Swiss Society of Pulmonology [34] but such statements are lacking, at this time, from leading North American Thoracic Societies. Data in uncomplicated influenza A infection, as well as a recent study with a younger, less symptomatic acute COVID-19 group suggest that even a minimally symptomatic population may suffer from reduced lung function and impaired gas transfer 1-2 months after acute illness [35, 36]. As such, we recommend lung function testing in all patients with acute COVID-19 seeking care for PASC (table 1). This may serve as a baseline to facilitate comparison if future lung disease were to develop.

\section{Imaging}

Radiographic features of acute COVID-19 infection have been a major area of research throughout the pandemic. Early studies focused on unique radiographic features and described findings of bilateral groundglass opacities, often peripherally located, affecting all lobes [37]. Interestingly, fibrotic parenchymal changes have been documented in the context of both subacute COVID-19 and chronic COVID-19-related respiratory failure. For example, 21 of 62 patients (33.9\%) with acute COVID-19 had computed tomography (CT) evidence of fibrotic changes that were more commonly discovered between 8 and 14 days than in under 7 days from symptom onset [37]. Findings compatible with fibrosis appear to develop early in the recovery process and even during protracted acute disease. Yu et al. [38] analysed CT scans of 32 patients during their acute infection and 9 days after discharge and showed that $44 \%$ of all these patients had parenchymal bands and $41 \%$ had irregular interface, consistent with early signs of fibrosis. One study of 24 patients who underwent chest CT scanning an average of 36 days after hospitalisation for COVID-19 infection demonstrated 62\% of patients with some evidence of pulmonary fibrosis [39]. Another study demonstrated abnormalities in 63\% of HRCTs at 2 months, of which $42 \%$ were ground glass and 19\% were fibrotic type lesions [40]. A single centre study reporting on survivors of moderate to severe COVID-19 during the first wave of the pandemic showed $72 \%$ of patients had

TABLE 1 Recommended screening for PASC-pulmonary fibrosis more than 1 month after acute COVID-19

\begin{tabular}{l} 
Severity of acute COVID-19 \\
\cline { 2 - 3 }
\end{tabular}


radiographic evidence of fibrotic-like changes (reticular pattern or honeycombing) at 6 months [41]. Figure 1 highlights the variety of findings described in cross-sectional imaging from our institutional experience with PASC.

In patients with respiratory PASC, MANDAL et al. [42] showed that 38\% of patients assessed at a median of 54 days post-discharge had chest radiographic abnormalities, with $9 \%$ of those having worsening radiographic abnormalities over the follow-up period. In a large cohort of patients from Wuhan, China, over $50 \%$ of patients who underwent CT scans 6 months after admission had abnormal radiographic findings; pulmonary interstitial changes, reported as ground-glass opacities and irregular lines, were the most common findings [4]. In patients with severe acute symptoms of COVID-19 evidence of radiographic fibrosis, bronchial dilatation, parenchymal bands and coarse subpleural reticulation without honeycombing are common [43].

The prospect that persistent or worsening radiographic abnormalities are missed on plain films, when compared with cross-sectional imaging, was underscored by D'CRUz et al. [44]. This group demonstrated that although $13 \%$ of patients in their study had evidence of COVID-19-related lung disease on chest radiographs, $46 \%$ of participants reported higher post-infection breathlessness scores, and $75 \%$ of those patients who went on to have CT scans were seen to have interstitial lung disease and/or airways disease [44].

Screening for pulmonary fibrosis in patients with persistent breathlessness after acute COVID-19 is currently dictated by a high index of suspicion, close medical follow-up and a combination of dedicated PFT and cross-sectional imaging. Plain radiographs and delayed follow-up may miss patients with persistent pulmonary morbidity. We agree, like SoLOMon et al. [45], that there should be standardisation with regard to CT appearances in PASC such as 1) predominantly ground glass; 2) predominantly fibrotic; and 3) mixed ground glass and fibrotic.

\section{Pathophysiology and mechanisms}

The underlying pathology of acute COVID-19 and PASC-pulmonary fibrosis has yet to be elucidated fully; however, there are relevant inferences that can be drawn from the literature on pulmonary fibrosis and recent pathobiologic mechanistic understanding during the current pandemic. Pulmonary fibrosis can occur in the setting of maladaptive resolution of lung injury or exaggeration of the reparative process [46]. Despite a myriad of initial pulmonary or extrapulmonary insults that result in ARDS, there may be a common downstream pathway that culminates in fibrosis. This pathway may be exacerbated by the production of cytokines from lung epithelial cells and macrophages or result from injurious mechanical ventilation [47, 48]. One recognised pathway in the distortion and remodelling of the pulmonary architecture relies on the fibroblast; a cell central to the fibrotic process [49]. Fibroblasts respond to
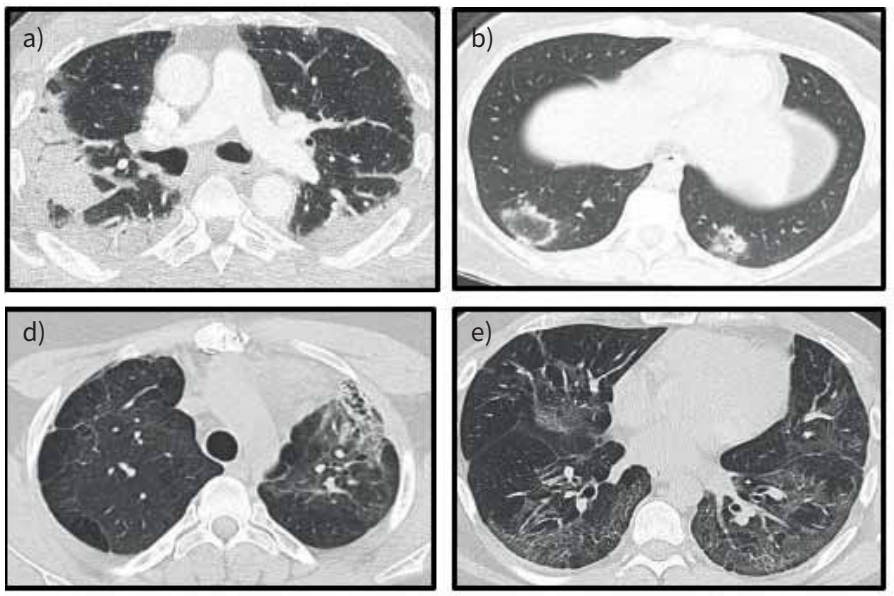
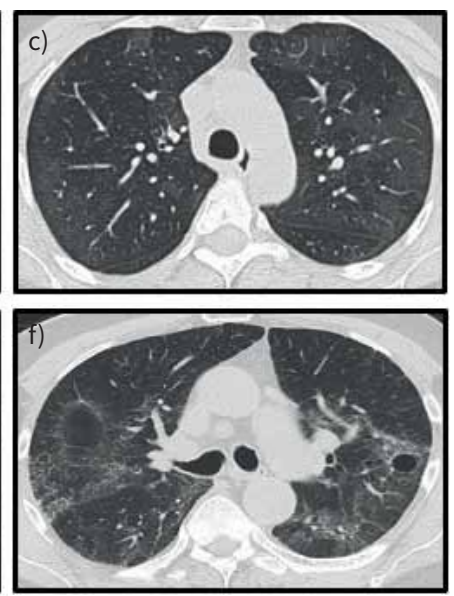

FIGURE 1 Radiographic features of PASC. Representative radiographic findings from CT scans collected at least 30 days after COVID-19 infection collected from the Post-COVID Registry at our centre. a) Dense consolidations. b) Patchy ground consolidation and ground glass with atoll sign. c) Diffuse ground-glass opacities. d) Fibrosis with honeycomb changes in the left upper lobe. e) Ground glass and reticular changes. f) Ground glass, reticulation and a pneumatocele. COVID-19: coronavirus disease 2019; CT: computed tomography; PASC: post-acute sequelae of COVID-19. 
alveolar injury, migrate to the alveolar space, secrete and regulate the extracellular matrix, and can differentiate into myofibroblasts [50]. Fibroblasts are sensitive to many inflammatory cytokines, notably interleukin (IL)-1 $\beta$, IL-6, transforming growth factor (TGF)- $\beta 1$; some of which are known to be upregulated during acute COVID-19 [51].

A second pathway in the fibrotic process relies on the unique roll that profibrotic monocyte-derived alveolar macrophages (MoAMs) play in pulmonary fibrosis [52, 53]. These macrophages stimulate and form reciprocal circuits with fibroblasts. Macrophages secrete platelet-derived growth factor A (PDGFA) to stimulate fibroblasts, and in turn, fibroblasts secrete macrophage colony-stimulating factor (M-CSF) to recruit further MoAMs. This process serves to maintain the circuit in an attempt to repair the epithelial

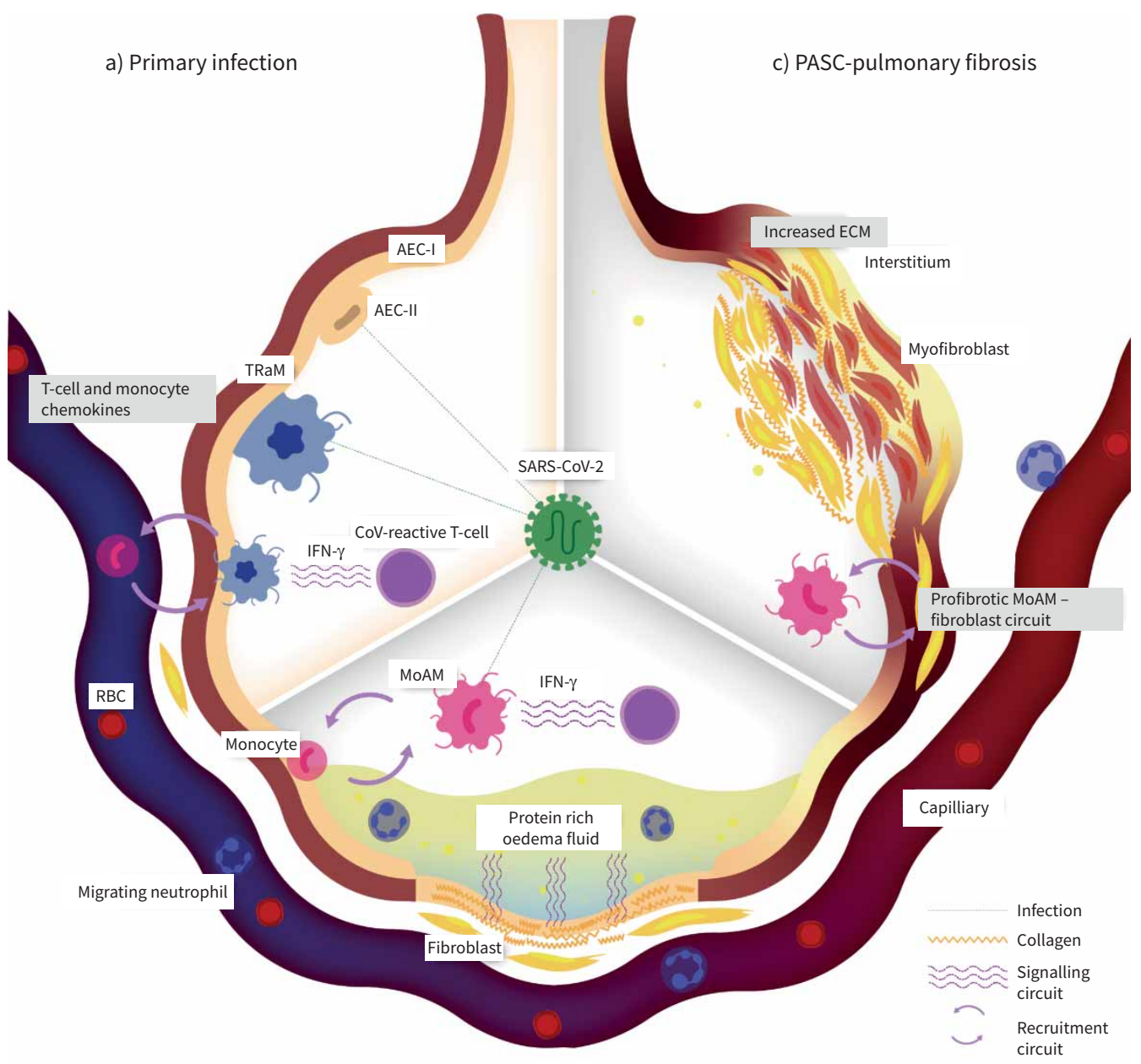

b) COVID-19 ARDS

FIGURE 2 Temporal unfolding of primary COVID-19 infection, COVID-19 ARDS and PASC-pulmonary fibrosis. a) SARS-CoV-2 infects AEC-IIs and TRaMs. Infected TRaMs express T-cell and monocyte chemokines. CoV-reactive T-cells recognise SARS-CoV-2 antigens and secrete IFN- $\gamma$. b) This results in death of TRaMs, proliferation of CoV-reactive T-cells and recruitment of monocytes, which become MoAMs. MoAMs become infected with SARS-CoV-2 and form a loop with CoV-reactive T-cells to create a replenishing circuit. c) Prolonged disease, duration of mechanical ventilation and exposure to higher level of cytokines transition the acute process of alveolar damage to fibroproliferative injury. Fibroblasts respond to the chronically elevated levels of cytokines to expand the ECM with matrix proteins. A circuit between MoAMs and fibroblasts may exist to promote fibrosis and differentiation of fibroblasts to myofibroblasts. AEC-I: type 1 alveolar epithelial cells; AEC-II: type 2 alveolar epithelial cells; ARDS: acute respiratory distress syndrome; COVID-19: coronavirus disease 2019; CoV-reactive T-cell: COVID-reactive T-cell; ECM: extracellular matrix; IFN: interferon; MoAM: monocyte-derived alveolar macrophage; PASC: post-acute sequelae of COVID-19; RBC: red blood cell; TRaM: tissue resident alveolar macrophages. 
injury [53]. Failed repair processes allow these signalling feedback loops to maladaptively persist, eventually filling the alveolar interstitium and alveoli with matrix proteins and fibroblasts (figure 2c).

PASC-pulmonary fibrosis may develop in patients who have cleared the virus and, as such, direct viral cytopathologic injury is unlikely to play a large pathobiologic role. However, rates of identified or often unidentified secondary pulmonary infections may play a role in injury and failure of the repair process resulting in increased risk of fibrosis. Rates of secondary pulmonary infections have been reported to range from $16 \%$ to $21 \%$ [54, 55]. Further, mechanisms that are responsible for acute lung injury may be responsible for chronic lung injury and maladaptive repair mechanisms leading to fibrosis. In acute COVID-19 respiratory failure, GRANT et al. [18] described autonomous circuits between infected tissue resident alveolar macrophages (TRaMs), MoAMs and T-cells, noting increased levels of genetic transcripts for interferon response genes, T-cell chemokines, and IL1 $\beta$ (figure $2 b$ ). These gene transcript levels have also been corroborated by increased levels of measured cytokines in acute COVID-19 including IL-1 interferon (IFN)- $\gamma$, IL-7-9, granulocyte-macrophage colony-stimulating factor, and tumour necrosis factor (TNF)- $\alpha$ [56]. Similar levels of cytokines were also reported in the prior SARS pandemic and do not

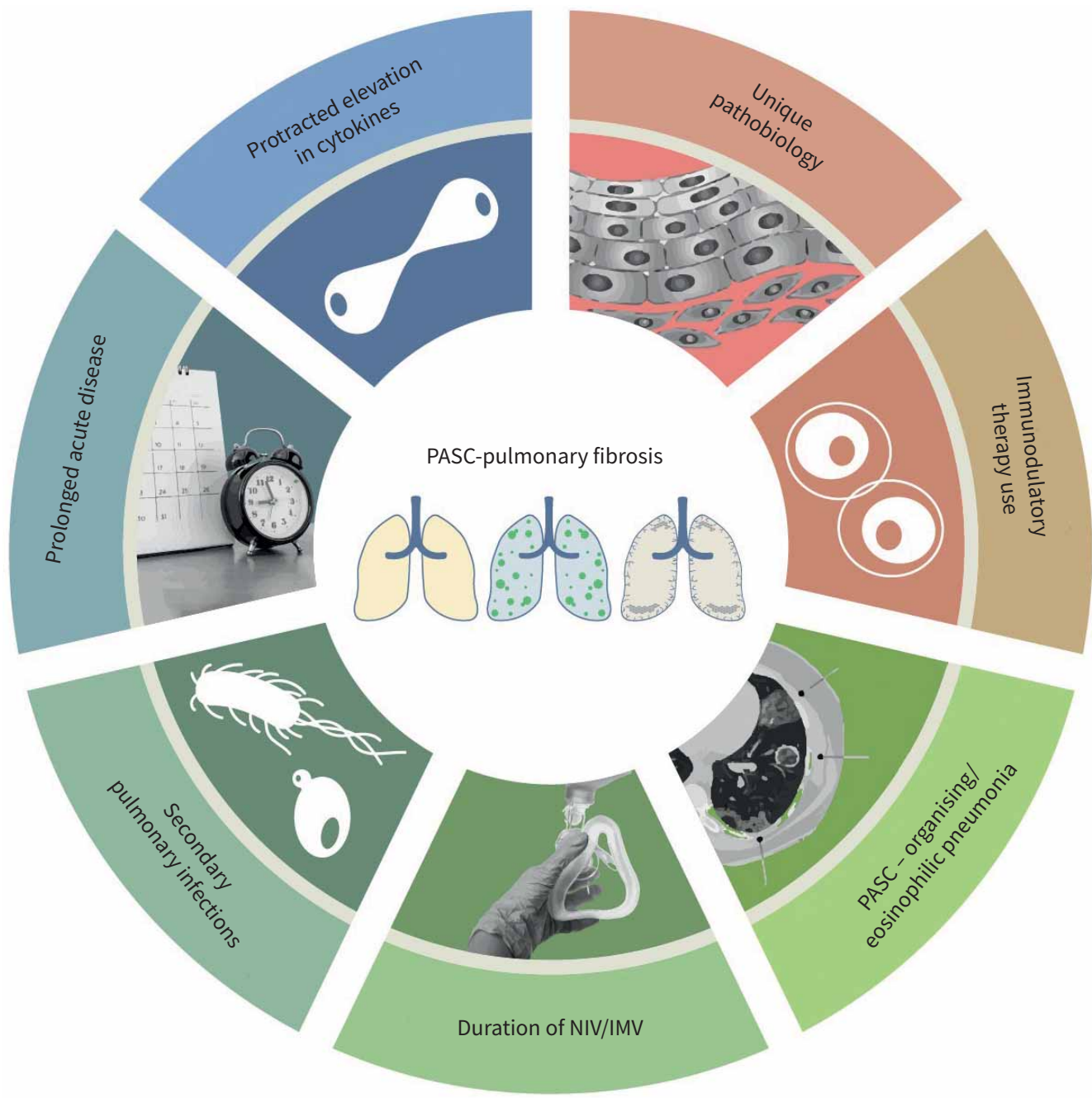

FIGURE 3 Avenues of future investigation into hallmarks of PASC-pulmonary fibrosis. Prospective future investigation should focus on the elucidating the contribution of unique pathobiologic factors (circuits between monocytes-T-cells, monocytes-fibroblasts), protracted elevations in cytokine levels, clinical factors (disease duration, NIV/IMV duration, secondary pneumonias) and use of adjunctive therapies for acute disease or PASC-organising pneumonia (immunomodulators such as glucocorticoids or anti-interleukin-6 antagonists) on the incidence, pathology and natural history of PASC-pulmonary fibrosis. IMV: invasive mechanical ventilation; NIV: noninvasive ventilation; PASC: post-acute sequelae of COVID-19. 
appear to be different from patients with influenza pneumonia [18, 57]. However, distinctive to the current SARS-CoV-2 pandemic may be the duration of the exposure to elevated levels of cytokines. BudingER et al. [58] postulate a prolonged phenotype of a slowly unfolding, spatially-limited inflammatory alveolitis. In this model, the prolonged exposure to elevated levels of cytokines is key in distinguishing COVID-19 pneumonia from influenza pneumonia, and may partly account for other manifestations of acute COVID-19. These pathobiologic findings are of significant interest in the understanding of PASC-pulmonary fibrosis. For example, if profibrotic monocytes interact through cytokine signalling with fibroblasts to promote fibrotic repair processes, the aforementioned circuit between T-cells and monocytes/ macrophages of acute COVID-19 may contribute to the fibroblastic response of PASC-pulmonary fibrosis. It is these circuits that may play a central role in modulating the lung response to injury and may be pivotal to understanding the pathobiology of PASC-pulmonary fibrosis (figure 2).

Altogether there is concern that the protracted nature of acute COVID-19, persistence of high levels of cytokines, monocyte/macrophage and T-cell circuits stimulating potential circuits between monocytes and fibroblasts, coupled with the extended duration of mechanical ventilation, could combine to promote a milieu in the alveoli with an increased likelihood of PASC-pulmonary fibrosis. An aspect of future investigation will be the study of biomarkers and the correlation with PASC-pulmonary fibrosis. Already, groups have identified serum and plasma levels of lipocalin-2, matrix metalloproteinase 7 , hepatocyte growth factor, Krebs von den Lungen-6, and leukocyte telomere length that track with abnormal imaging and are inversely correlated to PASC lung function [59-61]. Further research should include elucidating the contribution of relevant acute clinical risk factors of COVID-19 (i.e. duration of illness, receipt of invasive or noninvasive ventilation, use of corticosteroids, selective cytokine inhibitors, adjunct therapies, etc.) on the likelihood of PASC-pulmonary fibrosis (figure 3). For example, we have pursued respiratory PASC endotyping by integrating clinical data and objective quantitative CT scan analysis with flow cytometry, cytokine measurements and single-cell RNA sequencing of bronchoalveolar lavage fluid in an effort to develop prediction models for PASC-pulmonary fibrosis (figure 4).

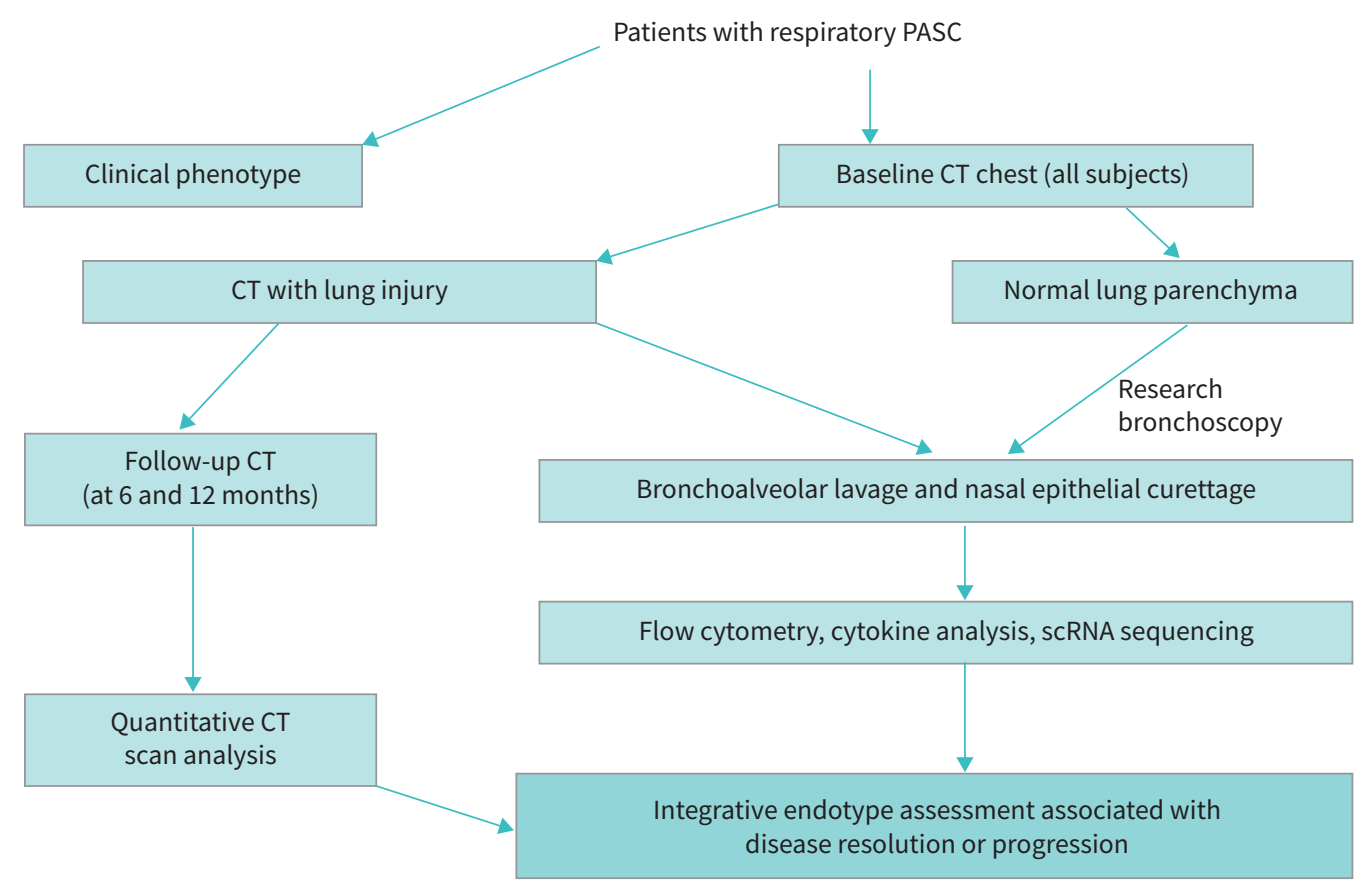

FIGURE 4 Our institutional protocol for endotyping the spectrum of respiratory PASC and prediction tools for PASC-pulmonary fibrosis. Patients referred for evaluation of respiratory PASC are phenotyped clinically and baseline cross-sectional images are obtained. Those with radiographic evidence of lung injury are offered bronchoscopy and nasal epithelial curettage. Those without radiographic lung injury are offered a research bronchoscopy as a control population. Follow-up cross-sectional images are obtained at pre-defined 6 month intervals and assessed quantitatively. Finally clinical, radiographic, and molecular data are integrated in an effort to study who will develop chronic lung diseases including PASC-pulmonary fibrosis. COVID-19: coronavirus disease 2019; CT: computed tomography; PASC: post-acute sequelae of COVID-19; scRNA: single-cell RNA sequencing. 


\section{Pathologic findings}

Pathologic specimens from patients surviving with PASC-pulmonary fibrosis have yet to be reported on but, as above, inferences can be drawn from autopsy series of patients with ARDS, pathologic specimens of patients with acute COVID-19, and patients with COVID-19 who underwent lung transplantation due to irreversible lung damage and fibrosis. In an autopsy series of 159 patients with non-COVID-19 ARDS, THILLE and colleagues [62] demonstrated that the longer the duration of illness the more likely the individual to have histologic signs of fibrosis. This group showed a striking $61 \%$ of patients with fibrotic histologic patterns when their disease duration was greater than 3 weeks. Whether the extent of acute injury or the duration of acute/subacute inflammation drove the fibrotic process remains to be understood. One study utilising bronchoscopic cryobiopsies of 12 patients with acute COVID-19 within 20 days of symptom onset showed epithelial and endothelial changes that were distinct and not reminiscent of either interstitial lung disease or diffuse alveolar damage [63]. In acute COVID-19, autopsy series of patients who died within 7-14 days describe exudative and proliferative diffuse alveolar damage (the histologic correlate to ARDS) [64-67]. In these series, which represent short durations of disease, there were minimal features of the fibrotic phase. A review of lung samples from 131 cases by PoLAK et al. [68] showed that while epithelial (85\%) and microvascular (59\%) changes were the main histologic patterns, fibrotic histologic patterns were found in $22 \%$ of cases. They noted that fibrotic histologic patterns were more common after 3 weeks of disease [68].

On the spectrum of acute to chronic COVID-19 disease, some patients may develop rapid, fulminant lung injury and fibrosis [69]. Histologic review of anatomic specimens after explantation for lung transplantation provide evidence of prolonged and extensive fibrotic lung injury. BHARAT et al. [70] demonstrated extensive injury and fibrosis in three patients undergoing lung transplantation for end-stage fibrosis secondary to COVID-19 (50-100 days after initiation of mechanical ventilation) and two post mortem autopsies in patients with COVID-19 who underwent prolonged mechanical ventilation (40 and 100 days after mechanical ventilation). Histologic and single-cell RNA sequencing were compared between these two groups and those with idiopathic pulmonary fibrosis undergoing lung transplantation. They showed similarities in gene expression profiles among those with end-stage COVID-19 and those with end-stage pulmonary fibrosis, suggesting a common pathobiologic mechanism [70].

\section{Role for antifibrotic medications}

There are two oral, commercially available antifibrotic medications to treat progressive pulmonary fibrotic conditions: pirfenidone and nintedanib. Both medications have been shown to slow the rate of decline of FVC by about $50 \%$, having some effects measurable by as early as $4-6$ weeks after initiation [71-73]. The use of antifibrotic therapy has extended beyond idiopathic pulmonary fibrosis to other forms of progressive pulmonary fibrotic processes, as was shown in the INBUILD study [63]. The roles for antifibrotic medication in both acute COVID-19 and PASC-pulmonary fibrosis remain to be determined and are being explored in ongoing clinical trials (for example, NCT04607928, NCT04619680, NCT04856111 and NCT04799743). In the acute COVID-19 setting, there is equivocal biological rationale for the use of antifibrotic medications: the prolonged nature of infection, the prevalence of early reticular changes, and the benefit of early initiation of antifibrotic medications on delayed FVC decline [74]. A small study in Japan of patients with acute COVID-19 requiring mechanical ventilation demonstrated that the use of nintedanib as compared with a historical COVID-19 control group resulted in reduced duration of mechanical ventilation and reduced percentages of high attenuation areas on CT at time of liberation from mechanical ventilation [75]. In the long term, the use of antifibrotic medications will need to be weighed against future evidence of whether the PASC-pulmonary fibrotic parenchymal process remains stable, becomes progressive or has a likelihood to improve.

\section{Impact of immunosuppression on fibrosis}

Currently, in acute COVID-19, glucocorticoids and IL-6 antagonists are therapies that have a positive impact on acute disease, but implications for PASC and pulmonary fibrosis remain unanswered. The initial UK-based RECOVERY trial, followed by several other randomised control trials and subsequent WHO-sponsored meta-analysis, demonstrated the benefit of glucocorticoid therapy in the management of hypoxaemic acute COVID-19 respiratory failure, with a benefit that correlates to a lower 28-day all-cause mortality [19, 76]. Similarly, a WHO-sponsored meta-analysis of 27 randomised control trials highlights a lower 28-day all-cause mortality in hospitalised patient's treated with IL-6 antagonists [77]. The effects that nonselective and selective immunosuppression with glucocorticoids and selective interleukin inhibition have on PASC-pulmonary fibrosis have not yet been described. It nevertheless remains plausible that with the use of immunomodulatory agents that result in decreased inflammation and decreased duration of mechanical ventilation, some of the risk for pulmonary fibrosis may be mitigated. 
A corollary to understanding immunomodulation on PASC-pulmonary fibrosis will be the study of PASC patients with organising pneumonia or eosinophilic pneumonia. A subgroup of patients with PASC have radiographic abnormalities consistent with organising pneumonia [78, 79]. It is not yet known how widespread this phenomenon is, but it has been described in patients with difficulty weaning from mechanical ventilation or with persistent hypoxaemia months after the acute infection [80]. These patients respond relatively well to glucocorticoid therapy, and it has been postulated that this phenomenon is under-recognised [81, 82]. It is unclear if the phenomenon of COVID-19-related organising pneumonia is mechanistically linked to the development of pulmonary fibrosis, and if treatment with corticosteroids may lessen the risk for PASC-pulmonary fibrosis, but these hypotheses deserve careful evaluation in future studies.

\section{Role for lung transplantation}

While extracorporeal membrane oxygenation (ECMO) has become the standard of care for select patients in the management in viral pneumonia with ARDS, lung transplant has historically been rarely performed in subacute post-viral fibrotic ARDS. Our centre first transplanted the lungs of a 29-year old with devastating COVID-19 lung destruction/fibrosis in June 2020 and has completed to date 29 lung transplants for patients with irreversible lung disease due to COVID-19 [69, 70]. The International Society for Heart and Lung Transplantation consensus guidelines do not make specific statements regarding patient selection in ARDS and outcomes are limited to multicentre experiences [83]. As a result, long-term outcomes for lung transplantation in ARDS are generally less studied than for chronic lung diseases [84, 85]. Further, there are several unique considerations that need to be taken into account [69]. Of particular note, there is uncertainty about the chances of meaningful lung function recovery for a given individual with PASC-pulmonary fibrosis. This area is in need of research to identify clinical or molecular tools to help prognosticate recovery. At present, the decision to pursue transplantation necessitates a judgement of the treating healthcare team about what constitutes an appropriate timeframe to evaluate patients for lung transplant.

Additionally, the need for deep sedation and often neuromuscular blockade can make participation in discussion about lung transplant with these patients difficult or impossible, even with the use of tracheostomy and ECMO to facilitate sedation weaning. Psychosocial evaluation is therefore dependent on collateral input and support from family and friends. In many cases, this population has not had close contact with the medical environment, so the abrupt life change of being post-transplant upon awakening from surgery is a psychologically difficult transition.

\section{Conclusion}

Pulmonary fibrosis may occur in a number of different clinical settings. Pulmonary fibrosis due to a SARS-CoV-2 infection can lead to considerable morbidity. Due to the global burden of COVID-19 and the virulence of SARS-CoV-2, pulmonary fibrosis will affect the lives of hundreds of thousands of patients within North America alone. Future studies are needed to clarify the natural history of this complication, describe its pathophysiology and assess the clinical and psychosocial impact on patients. Additional research avenues should include clinical and serologic phenotyping of patients with PASC-pulmonary fibrosis, with thorough epidemiologic identification of unique risk factors. Well-designed cohort studies and prospective clinical trials that enrol patients with acute COVID-19 and then follow these patients longitudinally over months and years after their infection are needed.

Clinicians around the world have developed multidisciplinary clinics to care for patients suffering from the sequelae of COVID-19 infection. It is crucial that these clinics collaborate to screen for and investigate PASC-pulmonary fibrosis. Biospecimen collection from patients at multiple time points as they recover from acute illness and progress to fibrosis will be the key to fully characterising the fibrotic process and may reveal targets for therapeutic interventions.

There are currently no proven therapeutic options for patients who develop PASC-pulmonary fibrosis. Clinicians, researchers and healthcare systems must meet this challenge urgently to minimise disability, maximise quality of life and prevent another global health disaster.

Provenance: Commissioned article, peer reviewed.

Members of the Northwestern Comprehensive COVID Center Consortium are as follows: Joe Bailey, Joseph Bass, Darren Brenner, GR. Scott Budinger, Mercedes Carnethon, Michael Cuttica, Charles Davidson, Eudora Eng, Matt Feinstein, Colin K. Franz, Daniel Ganger, Ray Goodwin, Linda Guthrie, Steve Hanauer, Fernanda Heitor, Michael Ison, Ravi Kalhan, Igor Koralnik, Gayle Kricke, Daniel Lee, Karlyn Martin, June McKoy, Janet Miller, Alexander Misharin, Malek El Muayed, Kannan Mutharasan, Ruben Mylvaganam, Cuong Nguyen, John Pandolfino, 
Jay Patel, Harris Perlman, Jayabalan Prakash, Susan Quaggin, Lisa Rosenthal, Elliot Roth, Eric Ruderman, Leslie Rydberg, Marc Sala, Robert Schleimer, Aneesha Shetty, Benjamin Singer, Anand Srivastava, Christian Stevoff, Jacob (Iasha) Sznajder, Esther Vorovich, John Wilkins, Lisa Wolfe, Richard Wunderink, Teresa Zembower, Lida Zheng.

Conflict of interest: R.J. Mylvaganam reports grant from Janssen Actelion Intelligence Award, outside the submitted work. J.I. Bailey has nothing to disclose. J.I. Sznajder has nothing to disclose. M.A. Sala reports grants or contracts from NIH - 5R01HL122477-07, CF Foundation - SALA18I0, and consulting fees from Medscape/WedMD, outside the submitted work.

Support statement: This work is supported in part by National Institute on Aging AG-049665, HL-154998 and T32HL076139. Funding information for this article has been deposited with the Crossref Funder Registry.

\section{References}

1 Office for National Statistics. The prevalence of long COVID symptoms and COVID-19 complications. www.ons.gov.uk/news/statementsandletters/theprevalenceoflongcovidsymptomsandcovid19complications Date last accessed: November 22, 2021. Date last updated: December 16, 2020.

2 Nalbandian A, Sehgal K, Gupta A, et al. Post-acute COVID-19 syndrome. Nat Med 2021; 27: 601-615.

3 Pijls BG, Jolani S, Atherley A, et al. Demographic risk factors for COVID-19 infection, severity, ICU admission and death: a meta-analysis of 59 studies. BMJ Open 2021; 11: e044640.

4 Huang C, Huang L, Wang Y, et al. 6-month consequences of COVID-19 in patients discharged from hospital: a cohort study. Lancet 2021; 397: 220-232.

5 Hui DS, Joynt GM, Wong KT, et al. Impact of severe acute respiratory syndrome (SARS) on pulmonary function, functional capacity and quality of life in a cohort of survivors. Thorax 2005; 60: 401-409.

6 Zhang P, Li J, Liu $\mathrm{H}$, et al. Long-term bone and lung consequences associated with hospital-acquired severe acute respiratory syndrome: a 15-year follow-up from a prospective cohort study. Bone Res 2020; 8: 8.

7 Das $\mathrm{KM}$, Lee $\mathrm{E}$, al Jawder $\mathrm{S}$, et al. Acute middle east respiratory syndrome coronavirus: temporal lung changes observed on the chest radiographs of 55 patients. AJR Am J Roentgenol 2015; 205: W267-W274.

8 Rabaan AA, Al-Ahmed SH, Haque S, et al. SARS-CoV-2, SARS-CoV, and MERS-CoV: a comparative overview. Infez Med 2020; 28: 174-184.

9 Lu R, Zhao X, Li J, et al. Genomic characterisation and epidemiology of 2019 novel coronavirus: implications for virus origins and receptor binding. Lancet 2020; 395: 565-574.

10 Hu B, Guo H, Zhou P, et al. Characteristics of SARS-CoV-2 and COVID-19. Nat Rev Microbiol 2020; 19: 141-154.

11 Masclans JR, Roca O, Muñoz X, et al. Quality of life, pulmonary function, and tomographic scan abnormalities after ARDS. Chest 2011; 139: 1340-1346.

12 Herridge MS, Cheung AM, Tansey CM, et al. One-year outcomes in survivors of the acute respiratory distress syndrome. N Engl J Med 2009; 348: 683-693.

13 Wilcox ME, Patsios D, Murphy G, et al. Radiologic outcomes at 5 years after severe ARDS. Chest 2013; 143: 920-926.

14 Burnham E, Hyzy R, Paine R, et al. Chest CT features are associated with poorer quality of life in acute lung injury survivors. Crit Care Med 2013; 41: 445-456.

15 Wu C, Chen X, Cai Y, et al. Risk factors associated with acute respiratory distress syndrome and death in patients with coronavirus disease 2019 pneumonia in Wuhan, China. JAMA Intern Med 2020; 180: 934-943.

16 Gattinoni L, Coppola S, Cressoni M, et al. COVID-19 does not lead to a "typical" acute respiratory distress syndrome. Am J Respir Crit Care Med 2020; 201: 1299-1300.

17 Schenck E, Hoffman K, Goyal P, et al. Respiratory mechanics and gas exchange in COVID-19-associated respiratory failure. Ann Am Thorac Soc 2020; 17: 158-1161.

18 Grant RA, Morales-Nebreda L, Markov NS, et al. Circuits between infected macrophages and T cells in SARS-CoV-2 pneumonia. Nature 2021; 590: 635-641.

19 RECOVERY Collaborative Group. Dexamethasone in hospitalized patients with COVID-19. N Engl J Med 2021; 384: 693-704.

20 Wu Z, McGoogan J. Characteristics of and important lessons from the coronavirus disease 2019 (COVID-19) outbreak in China: summary of a report of 72314 cases from the Chinese Center for Disease Control and Prevention. JAMA 2020; 323: 1239-1242.

21 Tmazini B, Maia I, Cavalcanti A, et al. Effect of dexamethasone on days alive and ventilator-free in patients with moderate or severe acute respiratory distress syndrome and COVID-19: the CoDEX randomized clinical trial. JAMA 2020; 324: 1307-1316.

22 Chopra V, Flanders SA, O'Malley M, et al. Sixty-day outcomes among patients hospitalized with COVID-19. Ann Intern Med 2021; 174: 576-578.

23 Carvalho-Schneider C. Follow-up of adults with noncritical COVID-19 two months after symptom onset. Clin Microbiol Infect 2021; 27: 258-263. 
24 Arnold DT, Hamilton FW, Milne A, et al. Patient outcomes after hospitalisation with COVID-19 and implications for follow-up: results from a prospective UK cohort. Thorax 2021; 76: 399-401.

25 Carfi A, Bernabei R, Landi F, et al. Persistent symptoms in patients after acute COVID-19. JAMA 2020; 324: 603-605.

26 Hui D, Wong K, Ko F, et al. The 1-year impact of severe acute respiratory syndrome on pulmonary function, exercise capacity, and quality of life in a cohort of survivors. Chest 2005; 128: 2247-2261.

27 Ngai J, Ko F, Ng S, et al. The long-term impact of severe acute respiratory syndrome on pulmonary function, exercise capacity and health status. Respirology 2010; 15: 543-550.

28 Park W, Jun K, Kim G, et al. Correlation between pneumonia severity and pulmonary complications in middle east respiratory syndrome. J Korean Med Sci 2018; 33: e169.

29 Guler SA, Ebner L, Beigelman C, et al. Pulmonary function and radiological features four months after COVID-19: first results from the national prospective observational Swiss COVID-19 lung study. Eur Respir J 2021; 57: 2003690.

30 Fumagalli A, Misuraca C, Bianchi A, et al. Pulmonary function in patients surviving to COVID-19 pneumonia. Infection 2021; 49: 153-157.

31 Mo X, Jian W, Su Z, et al. Abnormal pulmonary function in COVID-19 patients at time of hospital discharge. Eur Respir J 2020; 55: 2001217.

32 Torres-Castro R, Vasconcello-Castillo L, Alsina-Restoy X, et al. Respiratory function in patients post-infection by COVID-19: a systematic review and meta-analysis. Pulmonology 2021; 27: 328-337.

33 George PM, Barratt SL, Condliffe R, et al. Respiratory follow-up of patients with COVID-19 pneumonia. Thorax 2020; 75: 1009-1016.

34 Funke-Chambour M, Bridevaux P-O, Clarenbach CF, et al. Swiss recommendations for the follow-up and treatment of pulmonary long COVID. Respiration 2021; 100: 826-841.

35 Hall W, Douglas R, Hyde R, et al. Pulmonary mechanics after uncomplicated influenza A infection. Am Rev Respir Dis 1976; 113: 141-147.

36 Trinkmann F, Müller M, Reif A, et al. Residual symptoms and lower lung function in patients recovering from SARS-CoV-2 infection. Eur Respir J 2021; 57: 2003002

37 Zhou S, Wang Y, Zhu T, et al. CT features of coronavirus disease 2019 (COVID-19) pneumonia in 62 patients in Wuhan, China. AJR Am J Roentgenol 2020; 214: 1287-1294.

$38 \mathrm{Yu} \mathrm{M}, \mathrm{Li} \mathrm{Y,} \mathrm{Xu} \mathrm{D,} \mathrm{et} \mathrm{al.} \mathrm{Prediction} \mathrm{of} \mathrm{the} \mathrm{development} \mathrm{of} \mathrm{pulmonary} \mathrm{fibrosis} \mathrm{using} \mathrm{serial} \mathrm{thin-section} \mathrm{CT} \mathrm{and}$ clinical features in patients discharged after treatment for COVID-19 pneumonia. Korean J Radiol 2020; 21 : 746-755.

39 Antonio G, Wong K, Hui D, et al. Thin-section CT in patients with severe acute respiratory syndrome following hospital discharge: preliminary experience. Radiology 2003; 228: 810-815.

40 Writing Committee for the COMEBAC Study Group, Morin L, Savale L, et al. Four-month clinical status of a cohort of patients after hospitalization for COVID-19. JAMA 2021; 325: 1525-1534.

41 Caruso D, Guido G, Zerunian M, et al. Postacute sequelae of COVID-19 pneumonia: 6-month chest CT follow-up. Radiology 2021; 301: 210834.

42 Mandal S, Barnett J, Brill SE, et al. "Long-COVID": a cross-sectional study of persisting symptoms, biomarker and imaging abnormalities following hospitalisation for COVID-19. Thorax 2021; 76: 396-398.

43 Alarcón-Rodríguez J, Fernández-Velilla M, Ureña-Vacas A, et al. Radiological management and follow-up of post-COVID-19 patients. Radiologia 2021; 63: 258.

44 D'Cruz R, Waller M, Perrin F, et al. Chest radiography is a poor predictor of respiratory symptoms and functional impairment in survivors of severe COVID-19 pneumonia. ERJ Open Res 2021; 7: 00655-2020.

45 Solomon JJ, Heyman B, Ko JP, et al. CT of postacute lung complications of COVID-19. Radiology 2021; 301: 211396.

46 Burnham EL, Janssen WJ, Riches DWH, et al. The fibroproliferative response in acute respiratory distress syndrome: mechanisms and clinical significance. Eur Respir J 2014; 43: 276-85.

47 Pelosi P, Rocco PR. Effects of mechanical ventilation on the extracellular matrix. Intensive Care Med 2008; 34: 631-639.

48 Tremblay L, Miatto D, Hamid Q, et al. Injurious ventilation induces widespread pulmonary epithelial expression of tumor necrosis factor-alpha and interleukin-6 messenger RNA. Crit Care Med 2002; 30: 1693-1700.

49 Wijsenbeek M, Cottin V. Spectrum of fibrotic lung diseases. N Engl J Med 2020; 383: 958-968.

50 Flavell S, Hou T, Lax S, et al. Fibroblasts as novel therapeutic targets in chronic inflammation. Br J Pharmacol 2008; 153: Suppl. 1, S241-S246.

51 Vasarmidi E, Tsitoura E, Spandidos DA, et al. Pulmonary fibrosis in the aftermath of the Covid-19 era (Review). Exp Ther Med 2020; 20: 2557-2560.

52 Misharin AV, Morales-Nebreda L, Reyfman PA, et al. Monocyte-derived alveolar macrophages drive lung fibrosis and persist in the lung over the life span. J Exp Med 2017; 214: 2387-2404. 
53 Joshi N, Watanabe S, Verma R, et al. A spatially restricted fibrotic niche in pulmonary fibrosis is sustained by M-CSF/M-CSFR signaling in monocyte-derived alveolar macrophages. Eur Respir J 2019; 55: 1900646.

54 Chong WH, Saha BK, Ananthakrishnan R, et al. State-of-the-art review of secondary pulmonary infections in patients with COVID-19 pneumonia. Infection 2021; 49: 591-605.

55 Pickens CO, Gao CA, Cuttica MJ, et al. Bacterial superinfection pneumonia in patients mechanically ventilated for COVID-19 Pneumonia. Am J Respir Crit Care Med 2021; 204: 921-932.

56 Huang C, Wang Y, Li X, et al. Clinical features of patients infected with 2019 novel coronavirus in Wuhan, China. Lancet 2020; 395: 497-506.

57 Chien J, Hsueh P, Cheng W, et al. Temporal changes in cytokine/chemokine profiles and pulmonary involvement in severe acute respiratory syndrome. Respirology 2006; 11: 715-722.

58 Budinger GRS, Misharin AV, Ridge KM, et al. Distinctive features of severe SARS-CoV-2 pneumonia. J Clin Invest 2021; 131: e149412.

59 McGroder CF, Zhang D, Choudhury MA, et al. Pulmonary fibrosis 4 months after COVID-19 is associated with severity of illness and blood leucocyte telomere length. Thorax 2021; 76: 1242-1245.

60 Arnold DT, Donald C, Lyon M, et al. Krebs von den Lungen 6 (KL-6) as a marker for disease severity and persistent radiological abnormalities following COVID-19 infection at 12 weeks. PLOS ONE 2021; 16: e0249607.

61 Chun H, Coutavas E, Pine A, et al. Immunofibrotic drivers of impaired lung function in postacute sequelae of SARS-CoV-2 infection. JCl Insight 2021; 6: e148476.

62 Thille AW, Esteban A, Fernández-Segoviano P, et al. Chronology of histological lesions in acute respiratory distress syndrome with diffuse alveolar damage: a prospective cohort study of clinical autopsies. Lancet Respir Med 2013; 1: 395-401.

63 Doglioni C, Ravaglia C, Chilosi M, et al. Covid-19 interstitial pneumonia: histological and immunohistochemical features on cryobiopsies. Respiration 2021; 100: 488-498.

64 Schaller T, Hirschbühl K, Burkhardt K, et al. Postmortem examination of patients with COVID-19. JAMA 2020; 323: $2518-2520$

65 Ackermann M, Verleden SE, Kuehnel M, et al. Pulmonary vascular endothelialitis, thrombosis, and angiogenesis in COVID-19. N Engl J Med 2020; 383: 120-128.

66 Elsoukkary SS, Mostyka M, Dillard A, et al. Autopsy findings in 32 patients with COVID-19: a single-institution experience. Pathobiology 2021; 88: 56-68.

67 Carsana L, Sonzogni A, Nasr A, et al. Pulmonary post-mortem findings in a series of COVID-19 cases from northern Italy: a two-centre descriptive study. Lancet Infect Dis 2020; 20: 1135-1140.

68 Polak SB, van Gool IC, Cohen D, et al. A systematic review of pathological findings in COVID-19: a pathophysiological timeline and possible mechanisms of disease progression. Modern Pathol 2020; 33: 2128-2138.

69 Bharat A, Machuca T, Querrey M, et al. Early outcomes after lung transplantation for severe COVID-19: a series of the first consecutive cases from four countries. Lancet Respir Med 2021; 9: 487-497.

70 Bharat A, Querrey M, Markov NS, et al. Lung transplantation for patients with severe COVID-19. Sci Transl Med 2020; 12: 4282.

71 King TE Jr, Bradford WZ, Castro-Bernardini S, et al. A phase 3 trial of pirfenidone in patients with idiopathic pulmonary fibrosis. N Engl J Med 2014; 370: 2083-2092.

72 Flaherty KR, Wells AU, Cottin V, et al. Nintedanib in progressive fibrosing interstitial lung diseases. $N$ Engl J Med 2019; 381: 1718-1727.

73 Richeldi L, du Bois RM, Raghu G, et al. Efficacy and safety of nintedanib in idiopathic pulmonary fibrosis. N Engl J Med 2014; 370: 2071-2082.

74 George PM, Wells AU, Jenkins RG. Pulmonary fibrosis and COVID-19: the potential role for antifibrotic therapy. Lancet Respir Med 2020; 8: 807-815.

75 Umemura $\mathrm{Y}$, Mitsuyama $\mathrm{Y}$, Minami $\mathrm{K}$, et al. Efficacy and safety of nintedanib for pulmonary fibrosis in severe pneumonia induced by COVID-19: an interventional study. Int J Infect Dis 2021; 108: 454-460.

76 WHO Rapid Evidence Appraisal for COVID-19 Therapies (REACT) Working Group, Sterne JAC, Murthy S, et al. Association between administration of systemic corticosteroids and mortality among critically ill patients with COVID-19: a meta-analysis. JAMA 2020; 324: 1330-1341.

77 WHO Rapid Evidence Appraisal for COVID-19 Therapies (REACT) Working Group, Domingo P, Mur I, et al. Association between administration of IL-6 antagonists and mortality among patients hospitalized for COVID-19: a meta-analysis. JAMA 2021; 326: 499-518.

78 Lerum T, Aaløkken T, Brønstad E, et al. Dyspnoea, lung function and CT findings 3 months after hospital admission for COVID-19. Eur Respir J 2021; 57: 2003448.

79 Han X, Fan Y, Alwalid O, et al. Six-month follow-up chest CT findings after severe COVID-19 pneumonia. Radiology 2021; 299: E177-E186.

80 Vadász I, Husain-Syed F, Dorfmüller P, et al. Severe organising pneumonia following COVID-19. Thorax 2021; 76: 201-204. 
81 Kory P, Kanne JP. SARS-CoV-2 organising pneumonia: "Has there been a widespread failure to identify and treat this prevalent condition in COVID-19?" BMJ Open Respir Res 2020; 7: e000724.

82 Myall K, Mukherjee B, Castanheira A, et al. Persistent post-COVID-19 interstitial lung disease. An observational study of corticosteroid treatment. Ann Am Thoracic Soc 2021; 18: 799-806.

83 Weill D, Benden C, Corris P, et al. A consensus document for the selection of lung transplant candidates: 2014 - an update from the Pulmonary Transplantation Council of the International Society for Heart and Lung Transplantation. J Heart Lung Transplant 2015; 34: 1-15.

84 Chang $\mathrm{Y}$, Lee $\mathrm{S}$, Shim $\mathrm{T}$, et al. Lung transplantation as a therapeutic option in acute respiratory distress syndrome. Transplantation 2018; 102: 829-837.

85 Frick A, Gan C, Vos R, et al. Lung transplantation for acute respiratory distress syndrome: a multicenter experience. Am J Transplant 2021; in press. [https://doi.org/10.1111/ajt.16759]. 